\title{
Cette personne a quelque chose que je n'ai pas. Une approche contrastive de réactions du type de jalousie
}

\author{
Arkadiusz Koselak \\ Université Paul Verlaine de Metz, CELTED \\ a.koselak@laposte.net
}

L'être humain procède constamment à l'évaluation de situations qu'il perçoit. Une fois son évaluation faite, il choisit de réagir ou de ne pas réagir. Lorsqu'il choisit de réagir, il a plusieurs possibilités, le manque de réaction étant par ailleurs également une réaction. Un type de réponse possible est la réponse affective et c'est elle qui va nous intéresser ici.

A. Wierzbicka et J. Harkins (2001) postulent que les prédicats d'affect (émotions et sentiments) sont des «abréviations » des situations dans lesquelles ils s'actualisent. Autrement dit, une expression renvoyant à un affect cristallise, en quelque sorte, la perception et l'évaluation d'une situation par le locuteur. Tout comme le faisait déjà Fillmore (2003), on peut reconstruire ces situations notamment à travers la structure argumentale.

Dans cette présentation seront abordés les réactions de type «jalousie » dans une perspective contrastive. Les réactions de type « jalousie » ont en commun de s'intéresser à un bien (matériel ou non) que possède autrui et leur structure argumentale peut être formalisée de la manière suivante : X (Y possède $Z$ ). L'hypothèse de cette présentation est la suivante: compte tenu du fait que toutes les réactions en question partagent la même structure argumentale, les différences entre elles doivent se situer dans les rapports entre les différents arguments et autres éléments de la situation décrite. La structure argumentale étant relativement simple, les points de variations ne peuvent pas être très nombreux et il doit être possible de les schématiser sur la base des réactions étudiées. Je ne chercherai par contre pas à donner la description exhaustive de ces affects.

Le choix des concepts étudiés était guidé par les intuitions ${ }^{1}$ sur leur proximité. Il est possible de montrer en français l'interchangeabilité de l'envie et de la jalousie, ainsi que l'on peut le voir par exemple dans :

Il était jaloux, de son prestige et se vantait d'avoir été au service de deux ou trois princes de l'Europe. (DHÔTEL André/Le Pays où l'on n'arrive jamais/1955 Pages 100-101 / 6 UNE ÉTRANGE CROISIÈRE, Frantext)

Il lui enviait son prestige et se vantait d'avoir été au service de deux ou trois princes de l'Europe.

Le procédé de substition ne va cependant pas sans poser des problèmes. Il faut en effet décider si l'on cherche à tester les unités lexicales ou les images linguistiques ${ }^{2}$ de l'affect, ces dernières étant profilées par les différents lexèmes. Dans l'exemple supra il est procédé à la substitution de l'expression expérientielle qui désigne le ressenti vécu et cette substitution nécessite une réorganisation partielle de l'énoncé. Il est également possible de montrer les équivalences avec les autres langues par les procédés de traduction, ce qu'on peut voir dans les exemples de cette présentation.

La présentation se compose de trois parties. Premièrement, compte tenu du fait que la plupart des réactions étudiées sont des affects, je rappellerai le concept des affects tel qu'il a été élaboré dans la sémantique cognitive en général et dans l'approche wierzbickienne en particulier. Deuxièmement, j'examinerai les différentes réactions choisies en développant plus particulièrement les analyses des réactions françaises et 
polonaises et enfin, dans la troisième partie, je schématiserai les données obtenues. Le contenu sémantique des réactions étudiées sera expliqué à l'aide de la métalangue sémantique naturelle qui ne sera pas présentée ici. $^{3}$

\section{Les affects}

Les affects sont souvent considérés comme des états auxquels l'expérienceur est soumis passivement ( $c f$., par exemple, Flaux et Van de Velde, 2000). C'est l'héritage de la théorie cartésienne des passions. Or, actuellement, le terme passion n'est plus d'usage courant et apparaît lié aux théories philosophiques et aux pratiques des aliénistes ${ }^{4}$ (Plantin, 2003 : 111). Comme l'a montré Wierzbicka (1995), les termes génériques eux-mêmes véhiculent une conception du phénomène qu'ils désignent, on en déduit que les termes utilisés le plus fréquemment actuellement, émotion et sentiment, désignent une autre conception de la vie affective.

Les affects sont en effet des entités complexes. Comme l'ont montré les psychologues cognitifs Ortony, Clore et Collins (1988) les procès affectifs se composent de sous-processus cognitif, affectif et physique. Wierzbicka (par exemple : 1999) a montré à plusieurs reprises que cette complexité se trouve inscrite dans les langues et fait que l'on ne peut plus considérer les affects comme des états passifs entièrement indépendants de la volonté de l'expérienceur. Je vais préciser maintenant ce que l'on doit comprendre exactement comme composantes du processus affectif.

La composante cognitive est primordiale, c'est elle qui détermine si l'affect va avoir lieu ou non. Considérant que les affects sont des cristallisations de situations, dans la composante cognitive, l'expérienceur détermine s'il va réagir affectivement à la situation donnée. On peut appliquer ici le raisonnement d'Abelson (1981) concernant les scripts, pour qui une des conditions nécessaires pour que le script puisse avoir lieu est que l'individu doit décider d'entrer dans ce script. Le déclenchement d'un affect dépend toujours de l'expérienceur même si le phénomène d'appraisal [évaluation] qui est en jeu dans la composante cognitive n'est pas nécessairement conscient. L'expérienceur doit procéder à une évaluation axiologique de la situation : il doit décider si la situation est neutre, bonne ou mauvaise pour lui. Bien évidemment cette évaluation de la situation est complexe et s'appuie sur plusieurs paramètres de la situation. Ces paramètres varient selon les affects et ils seront abordés lors de l'analyse de chaque affect.

Une fois cette évaluation faite, peut s'activer une tendance à l'action (vouloir quelque chose). Simultanément, s'active la composante affective (ressentir quelque chose de bon ou mauvais) et, mais pas toujours, la composante physique. Cette dernière comporte deux types de phénomènes: le ressenti physique corporel indépendant de la volonté de l'expérienceur (il se passe quelque chose dans mon corps) et les comportements (je fais quelque chose avec mon corps). La composante physique, dont je ne m'occuperai pas ici, est non spécifique. Les analyses des équivalents de la peur en anglais et en russe qu'effectue Apresjan (2000) montrent que cet affect peut se caractériser par des comportements contradictoires (fuir et être figé). Les composantes cognitive et physique sont indissociables du ressenti ; le ressenti bon ou mauvais, sans ces deux composantes, est indéfinissable et n'est pas encore un affect, c'est la configuration de ces trois composantes qui est spécifique pour un affect.

En dehors de ces trois composantes des affects il est nécessaire d'étudier leur structure argumentale qui découle de la composante cognitive. Il est utile dans ce travail d'introduire une distinction entre la structure actancielle et la structure argumentale: la structure argumentale est de nature sémantique et concerne les arguments conceptuels d'un procès. Ces arguments inhérents se réalisent ou non dans la structure actancielle des énoncés; la non-réalisation d'un argument en tant qu'actant étant en soi signifiante. 


\section{Quelques concepts - réactions à la situation « cette personne a quelque chose "}

\subsection{La jalousie}

Le concept de jalousie français concerne deux affects différents bien que nécessairement liés, l'un concerne quelque chose qu'un autre possède et que l'expérienceur n'a pas et l'autre s'actualise dans une situation dans lequel l'expérienceur a quelque chose et risque de le perdre. On observe que les expressions de jalousie en français contemporain ont tendance à se spécialiser pour exprimer l'un et l'autre affect, mais il ne s'agit que d'une tendance. Ainsi l'adverbe jalousement concerne plutôt la situation dans laquelle l'expérienceur a quelque chose et risque de le perdre, comme en témoigne la collocation privilégiée garder jalousement (les secrets). L'expression essive être jaloux en emploi absolu tout comme être jaloux de $N$ (humain particulier lié à l'expérienceur) concernent la jalousie que l'on peut appeler conjugale. Les expressions de jalousie conjugale sont nécessairement liées au sens qui nous intéresse, c'est-à-dire à la convoitise d'un objet, mais je ne vais pas rechercher ici ce lien et je me contenterai d'analyser plus en avant la convoitise d'un objet ${ }^{5}$. Enfin le verbe jalouser est devenu rare et il n'en sera pas question ici.

La jalousie se construit sur le schéma argumental : X (Y possède $\mathrm{Z})$. La formulation 'Y possède Z' n'est pas à prendre au sens uniquement matériel, on peut être jaloux de la voiture de son voisin tout comme on peut être jaloux de la popularité de quelqu'un. Wierzbicka (1999: 98) utilise une formulation plus neutre pour décrire envy anglaise « quelque chose de bon est arrivé à cette personne » mais cette formulation a l'inconvénient de présenter Y comme n'étant pas responsable de la situation, or on peut être jaloux de la réussite de quelqu'un. Dans la mesure où le paramètre de la responsabilité de $\mathrm{Y}$ dans la situation Y possède $\mathrm{Z}$ n'a pas encore été suffisamment étudié, j'utiliserai la formulation neutre de ce point de vue. On peut également penser que la possession au sens matériel est plus primaire dans le cas de la jalousie.

L'expérienceur de la jalousie n'envisage pas Y et Z séparément, un énoncé comme:

Je suis jaloux de lui.

compris au sens de convoitise, est sémantiquement incomplet, tout comme :

?? Je suis jaloux de la grande maison.

Par contre cet énoncé devient complet si on y introduit $\mathrm{Y}$ :

Je suis jaloux de sa grande maison.

Observons encore cet énoncé :

En fait je suis jaloux de la génération de mes parents et de mes grands parents. Ils ont tout eu : maison, voiture, sécurité de l'emploi. (internet)

Le premier énoncé est incomplet sans le deuxième : il ne comprend que Y. Z est explicité dans le deuxième énoncé.

Les rapports entre $\mathrm{X}$ et $\mathrm{Z}$ sont très simples : $\mathrm{X}$ n'a pas $\mathrm{Z}$ et veux avoir un objet du type de $\mathrm{Z}$. Les rapports entre $\mathrm{X}$ et $\mathrm{Y}$ semblent un peu plus complexes. Les énoncés avec, comme l'objet de jalousie, un individu différent de nous d'une ou d'autre façon, semblent pour le moins bizarres :

? Je suis jaloux du président de la république car il peut voyager gratuitement.

La question qui se pose ici est de savoir si la contrainte qui semble se dessiner porte plutôt sur l'individu Y ou sur l'objet Z. Autrement dit, s'il s'agit d'une nécessité d'appartenance de Y à la même classe que 
l'expérienceur ou si l'objet doit être accessible à l'expérienceur. Il s'agirait plutôt de la deuxième solution, l'énoncé suivant ne semble pas poser de problème :

Je suis jaloux du pape, il parle tellement de langues. ${ }^{6}$

Y n'est donc pas contraint, par contre une contrainte supplémentaire de Z apparait : il doit être accessible à l'expérienceur, par exemple l'idée de voyager gratuitement ne m'est pas théoriquement accessible alors que je peux apprendre beaucoup de langues.

On trouve dans les dictionnaires une idée d'énergie négative dirigée envers l'objet Y, par exemple Le Petit Robert parle d' « hostilité ». Cette « hostilité » n'est visible que très rarement dans les énoncés et on peut se demander si elle est linguistiquement pertinente, c'est-à-dire si elle fait partie de l'image linguistique du monde. La jalousie est certes négative, mais son orientation négative est plutôt liée à l'expérienceur lui-même. On peut dire que l'expérienceur, en évaluant la situation (Y possède Z), ressent quelque chose de mauvais, parfois ce ressenti s'apparente à une maladie, comme en témoignent les expressions vert de jalousie où la couleur verte métaphorise le processus morbide et, plus littéralement, la jalousie maladive.

On peut expliquer la jalousie de la manière suivante :

Xpense :

cette personne (Y) a une chose (Z)

je pense que je pourrais avoir la chose (Z)

je n'ai pas cette chose (Z)

je voudrais avoir cette chose (Z)

à cause de cela $\mathrm{X}$ ressent quelque chose de mauvais ${ }^{7}$

Résumons-nous. Lorsque la jalousie est exprimée par la structure essive être jaloux en emploi absolu, elle est généralement interprétée comme une qualité stable d'un individu. L'actant Y peut apparaître tout seul sur la surface de l'énoncé mais alors l'actant Z (possession de Y) doit être saillant dans la co(n)texte, sinon l'expression sera interprétée en terme de jalousie conjugale. L'objet $Z$ ne peut jamais apparaître tout seul ce qui montre bien que cet objet ne peut être convoité qu'en tant que possédé par Y.

\subsection{L'envie}

L'envie est proche de jalousie et les locuteurs du français ne sont souvent pas capables de les différencier métalinguistiquement. L'envie est polysémique et l'une de ses significations appartient au domaine de la volition (avoir envie de). L'envie semble être complexe de point de vue axiologique. Elle fait partie des sept péchés capitaux de la religion catholique, mais les locuteurs contemporains pensent que la jalousie est plus négative que l'envie. Le jugement axiologique change lorsqu'on aborde l'adjectif envieux qui est très péjoratif.

Il semble que, contrairement à ce que disent les dictionnaires, l'envie ne contient pas, du moins pas nécessairement, du ressenti négatif et, ce qui s'en suit, elle n'est probablement pas un affect! L'envie apparaît actuellement en effet comme une forme très «légère » de jalousie. L'expérienceur de l'envie ne veut pas l'objet possédé par l'autre, il voudrait l'avoir, l'idée de ne pas avoir ce que possède l'autre ne provoque pas de ressenti négatif. Comme elle ne provoque pas de ressenti positif non plus, il ne peut pas s'agir d'un affect, les affects neutres n'existant pas (MacLean, 1980). On peut penser que l'envie était un affect, très négatif du point 
de vue doxal, mais elle ne l'est plus ${ }^{8}$. L'envie appartiendrait donc au domaine du vouloir. Mais pourquoi alors aurait-on besoin de deux expressions ayant envie comme base : envier et avoir envie ? Un détour par le domaine de la volition s'impose. Le primitif de ce domaine est le verbe vouloir. Il désigne une volonté consciente de l'expérienceur, autrement dit une participation sinon une décision. L'expression avoir envie, décrit une tension vers quelque chose sans que l'expérienceur se présente comme responsable :

Je veux une glace $=$ j'ai décidé que je voulais une glace

J'ai envie d'une glace = il y a quelque chose en moi qui fait que je veux une glace

Cela semble d'autant plus évident avec des expressions comme faire pipi ou encore dormir :

$J^{\prime}$ ai envie de faire pipi $=$ il y a une pression en moi (dans ma vessie)

Je veux faire pipi = je veux le faire maintenant

$J^{\prime}$ ai envie de dormir $=$ je sens que le sommeil vient

Je veux dormir $=$ je veux me coucher

Dans le cas du verbe envier, il faut que l'objet en question appartienne à quelqu'un. C'est dans cela que l'envie se rapproche de la jalousie, l'objet de l'envie n'est pas un quelconque objet mais un objet possédé par quelqu'un. Le verbe envier accepte deux schémas actanciels : envier quelqu'un et envier quelque chose à quelqu'un. Le premier de ces deux schémas laisserait entendre que $\mathrm{Y}$ et $\mathrm{Z}$ ne sont pas si intimement liés comme c'était le cas de la jalousie. L'observation des énoncés permet de se rendre rapidement compte que même si Z n'est pas formellement lié à Y, il est présent soit dans l'énoncé, comme c'est le cas de:

Maman, hier une collègue a perdu sa mère, je suis allée à l'enterrement. Ma collègue pleurait beaucoup, mais moi je l'enviais, elle l'a vue mourir, elle l'a lavée, parée pour le départ éternel. (internet)

soit dans son entourage:

Quelque part, au fond de moi, je l'enviais. Il maniait l'épée comme si c'était son épouse.(internet)

La structure envier quelqu'un fonctionne donc comme la structure être jaloux de quelqu'un. Par contre la deuxième structure possible envier quelque chose à quelqu'un est spécifique à l'envie et cette construction contient un datif. Bien évidemment dans la mesure où il y a dans cette structure deux objets, l'un est nécessairement un objet indirect, on peut conclure qu'il s'agit uniquement d'un cas structurel. Cependant, même si le datif est là pour une raison structurelle, il n'en véhicule pas moins une information sémantique, et déterminer la nature de ce datif serait peut-être utile pour comprendre la spécificité de l'envie. On ne peut pas dire que le datif, dans le cas de l'envie, désigne une quelconque idée d'affection de $\mathrm{Z}$, on ne peut pas non plus parler du datif éthique. Mais par contre on peut poser l'hypothèse qu'il s'agit du datif de possession inaliénable. Il semblerait en effet que ce qui est le véritable l'objet de l'envie c'est la situation de Y, autrement dit l'expérienceur de l'envie ne souhaiterait pas tellement posséder $Z$ mais être à la place de $Y$, dans la mesure où $Z$ est une partie intégrante de $Y$. Il en découle qu'il n'y a aucune contrainte ni sur Y ni sur Z puisqu'il n'est pas question d'accéder à $Z$, mais juste de penser que ce serait bien d'être dans la même situation que Y sans qu'il y ait un mauvais ressenti à cause de cela. Dans les énoncés que nous avons observés, l'envie porte plus souvent sur les qualités, traits de caractères, réussite, etc. que sur des objets matériels, donc sur les phénomènes que l'on peut considérer comme inaliénables. Lorsqu'il s'agit des objets matériels, comme par exemple dans :

Je lui envie son bonnet. (internet)

il ne s'agit pas tellement de vouloir le bonnet de Y, comme nous le renseigne le contexte, mais d'avoir chaud comme Y l'a. Sans aucune animosité par ailleurs. 
Il n'est pas question ici de résoudre la problématique de l'envie. Pour la comprendre, il faudrait étudier l'histoire de ce concept. Il nous suffit de dire que l'envie a exactement la même structure argumentale que la jalousie. Par contre, il ne s'agit pas d'un affect. Et il n'y a aucune contrainte ni sur Z ni sur Y.

On pourrait expliquer l'envie ainsi :

Xpense :

quelque chose de bon (Z) est arrivé à cette personne (Y)

je voudrais que les choses comme cela m'arrivent

si les choses comme cela m'arrivent je ressentirai quelque chose de bon

On peut également avoir recours à la théorie des mondes à la manière que l'utilise Zalizniak (1991) pour expliquer le concept de regret. Dans le monde M1, actuel, je perçois la situation (Y possède $\mathrm{Z}$ ), dans le monde projeté $\mathrm{M} 2$ je possède $\mathrm{Z}$ et je ressens quelque chose de bon. Dans le monde projeté on peut parler alors d'un affect positif, mais ce n'est plus de l'envie.

\subsection{Zazdrość}

Zazdrość polonaise ${ }^{9}$ semble identique à la jalousie française. Gugała (2004) relève les valeurs identiques au français : elle peut signifier tout aussi bien une convoitise du bien d'autrui, une crainte au sujet de l'être aimé et une crainte de perdre l'exclusivité d'un objet. Au plan formel, contrairement au français, le verbe zazdrościć [jalouser] est vivant et sert de l'expression expérientielle de base. La structure actancielle de ce verbe est identique à celle de l'envie (et non celle de la jalousie) : Y qui possède l'objet $\mathrm{Z}$ est au datif, comme par exemple dans :

Zazdrościłam koleżankom, że ich rodzice się kochaja, że chodzq razem na spacery, że się wspólnie śmieja itd. (Internet)

[j'étais jalouse de mes copines:DAT, que leurs parents s'aimaient, qu'ils allaient se promener ensemble, qu'ils riaient ensemble, etc.]

$\mathrm{Z}$ est contraint de la même manière que dans la jalousie.

Contrairement à la jalousie mais tout comme l'envie, zazdrość possède des expressions qui servent à valoriser des objets godny pozazdroszczenia [digne d'être jalousé = enviable] qui peut également servir à valoriser un objet négativement dans la collocation figée nie do pozazdroszczenia [approximativement : pas enviable].

Malgré ces quelques différences avec la jalousie, zazdrość fonctionne comme cet affect. Il focalise principalement l'objet possédé par quelqu'un et la volonté de l'expérienceur à posséder cet objet. L'explication verbale donnée pour la jalousie vaut donc également $\mathrm{ici}^{10}$.

\subsection{Zawiśćc ${ }^{11}$}

Zawiść est un affect que les Polonais s'auto-attribuent souvent comme en témoignent les collocations fréquentes du type (typowa) polska zawiść [(typique) polonaise zawiść]. Et ceci même s'il s'agit d'un affect très négatif du point de vue de l'axiologie externe : ressentir quelque chose comme ceci est très mauvais. Tellement mauvais que l'on ne trouve pas d'expressions de cet affect actualisées à la première personne. 
Zawiść est un affect que l'on peut qualifier de particulièrement pervers : contrairement à la zazdrość ou à la jalousie, l'expérienceur de zawiść ne souhaite pas avoir l'objet que l'autre possède mais la disparition de cet objet. La collocation fréquante bezinteresowna zawiść [zawiść non intéréssée] en témoigne. La volonté de la destruction relie zawiść aux affect du type de la haine.

Avant de poursuivre, pour faciliter au lecteur non polonophone la compréhension de cet affect, voici son explication verbale :

\author{
Xpense : \\ quelque chose de bon (Z) est arrivé à cette personne (Y) \\ je pense que c'est mauvais \\ je veux ceci : quelque chose de mauvais arrive à cette personne \\ (je veux que cette personne n'ait plus Z) » \\ à cause de cela X ressent quelque chose de mauvais
}

Reformulons : l'expérienceur, selon son système de croyances, considère que la situation (Y possède Z) n'est pas normale, il veut que la situation redevienne normale et elle ne peut le devenir que si Y ne possède plus $Z$. Dans les énoncés étudiés, il s'agit le plus souvent de vouloir la disparition de l'objet Z, mais, dans quelque rares cas plus extrêmes, il s'agit de souhaiter la disparition de Y. Dans tous les cas, il ne s'agit que d'une volonté d'un souhait, l'expérienceur ne passe pas à l'action, ce qui est propre à tous les affects si l'on en croit la philosophie analytique (Church, 1995) et l'éthologie (Eibl-Eibesfeldt, 1996), ces deux disciplines considérant que les affects sont des actions intériorisées.

La structure argumentale de zawiść est identique à celles des autres réactions qui sont étudiées ici. Linguistiquement, zawiść est exprimée en polonais contemporain soit par l'adjectif attributif zawistny soit par l'adverbe zawistnie. Le verbe zawiścić a existé mais il est sorti d'usage au cours du XXe siècle, probablement à cause de l'axiologie négative de zawiść. Comme cela a été déjà mentionné, l'adjectif et l'adverbe sont généralement hétéroattribués. Dans l'énoncé infra on observe un discours à la première personne qui nie qu'il s'agisse de zawiśćc cependant cet affect est identifié par l'interlocuteur. Zawiść est trop négative pour que l'on puisse admettre la ressentir. Compte tenu du manque de l'expression expérientielle, les arguments $\mathrm{Y}$ et $\mathrm{Z}$ ne sont pas formellement liés à la situation de zawiść mais doivent être récupérables dans le co(n)texte.

L'expérienceur de zawiść n'a pas besoin de connaître Y, il n'est pas non plus nécessairement égal à Y comme en témoigne l'énoncé suivant :

Oczywiście jak wiele Polaków jesteś mocno zawistny. [...] - Nie jestem zawistny, tylko uważam, że w tak biednym mieście taka wystawność jest nie na miejscu. (internet)

[- bien sûr tu es très envieux (zawistny) comme beaucoup d'autres Polonais. [..] - Je ne suis pas envieux (zawistny), seulement je considère qu'une telle manifestation de luxe n'a pas lieu d'être dans une ville si pauvre]

Dans cet énoncé le locuteur-expérienceur se positionne face à la politique municipale, il n'y a donc aucune égalité envisageable. Autrement dit il n'y a pas de restriction sur l'objet Y. Quant aux rapports de X à Z, l'expérienceur ne convoite pas $\mathrm{Z}$, ou en tout cas, cette convoitise ne fait pas partie de zawiść. Il est cependant possible qu'une telle convoitise provoque zawiść. 


\subsection{Schadenfreude}

Schadenfreude allemand est un composé transparent, Schaden signifiant «dommage, dégât » et Freude la joie. Schadenfreude est traduit par les lexicographes par «joie maligne, malin plaisir, plaisir sadique » (Harrap's Universal. Dictionnaire Français-Allemand/Allemand-Français). Il s'agit donc d'un affect à part dans le groupe qui nous intéresse ici, il est positif pour l'expérienceur et l'on peut le schématiser ainsi :

\section{T1: X perçoit la situation (Y possède $\mathrm{Z}$ )}

$\mathrm{X}$ pense quelque chose de mauvais de cette situation (il semble impossible de décider ici si l'expérienceur ressent quelque chose de mauvais, un affect comme zawiść ou la jalousie par exemple ${ }^{12}$ );

$\mathrm{T} 2$ : quelque chose de mauvais arrive à $\mathrm{Y}$ (mais $\mathrm{Y}$ ne perd pas nécessairement $\mathrm{Z}$ ), $\mathrm{X}$ pense et ressent quelque chose de bon, X ressent Schadenfreude.

L'axiologie externe de Schadenfreude est toujours négative et c'est probablement pour cette raison, tout comme c'était le cas de zawiść, qu'on ne trouve pas de verbe qui marquerait nécessairement un certain engagement de l'expérienceur mais les expressions die Schadenfreude spüren [ressentir Schadenfreude] ou schadenfroh sein [être schadenfroh], les deux étant rares à la première personne pour la même raison. Ces deux expressions ne nécessitent pas la mention d'objet, cette mention ne paraît pas nécessaire dans la mesure où il n'est question ni d'un objet précis ni de la perte d'un objet précis. Pour Wierzbicka (1999), Y doit nécessairement être plus ou moins égal à $\mathrm{X}$ pour que Schadenfreude puisse être actualisée.

Wierzbicka (1999 : 104) explique le concept de Schadenfreude de la manière suivante :

Xpense :

beaucoup de bonnes choses sont arrivées à cette personne

cette personne a pensé : 'c'est bon'

quelque chose de mauvais est arrivé à cette personne maintenant

je pense : c'est bon

à cause de cela X ressent quelque chose de bon

Zawiść polonaise et Schadenfreude se ressemblent. On pourrait dire que la différence entre les deux affects est, d'une certaine manière, une différence de modalité, zawiść est une volonté qu'un événement arrive, Schadenfreude est une réaction au même type d'évènement déjà arrivé. Zawiść est un affect négatif à tous les niveaux axiologiques, alors que Schadenfreude est positif au niveau de l'axiologie interne : l'expérienceur pense et ressent quelque chose de bon. Tandis que zawiść a plutôt tendance à viser un objet précis, Schadenfreude est une réaction à n'importe quel type de malheur arrivé à Y. Il y a par ailleurs, dans Schadenfreude une contrainte d'égalité entre $\mathrm{X}$ et $\mathrm{Y}$ alors que zawiść n'est pas contrainte de ce point de vue. 


\subsection{Avund et missunsamhet ${ }^{13}$}

Les affects suédois sont traités ensemble à cause de leur étymologie commune, morphologiquement il s'agit pratiquement de doublets. Les deux lexèmes sont construits sur la base du verbe unna [approximativement: aimer, souhaiter du bonheur] ${ }^{14}$ et les préfixes $a v$ - et miss- [l'idée de contraire]. Dans le suédois moderne avund et missunsamhet se sont spécialisés même s'ils restent toujours proches.

Je commencerai par un bref détour par le verbe unna, il est indispensable à la compréhension des concepts qui nous intéressent et ceci pas uniquement parce qu'il constitue la base étymologique, mais également parce qu'il reflète certains aspects de la culture suédoise et scandinave en général ${ }^{15}$. En suédois moderne, le verbe unna signifie approximativement accorder (quelque chose à quelqu'un), comme dans :

Jag skall unna mig ett par nya skor.

[je vais m'accorder une paire de nouvelles chaussures]

mais ce verbe véhicule plus d'informations, on l'utilise en effet pour signifier également que « compte tenu de circonstances, je ne devrais peut-être pas le faire » et ce verbe sous-entend que ce que l'on s'accorde, on le fait pour son plaisir. Cette notion de plaisir auto-accordé n'est pas nécessairement très positive dans l'ambiance du protestantisme plutôt austère. Par contre la traduction s'avère plus difficile lorsque l'expérienceur et l'objet humain Y sont disjoints :

Vilken missunsamhet! Jag har betalat hela bilen själv och hon unnar inte mig den!

[Quel jalousie ${ }^{16}$ ! J'ai payé ma voiture tout seul et elle ne me l'accorde pas!]

Dans cet énoncé il faut lire quelque chose comme : « elle ne croit pas ça possible (certainement parce qu'elle ne peut pas faire cela elle-même et si elle ne peut pas, je ne devrais pas pouvoir moi non plus ». On peut y avoir l'allusion à un certain égalitarisme qui se confirme par le concept de missunsamhet.

Mais commençons par l'avund. Ce concept semble correspondre directement à la jalousie française et à zazdrość polonaise mais il ne concerne que la convoitise de quelque chose, le suédois possède en effet un autre mot pour désigner la jalousie conjugale : svartsjuka. L'objet $\mathrm{Z}$ de $\mathrm{Y}$ doit être théoriquement accessible à l'expérienceur.

En ce qui concerne l'expression de l'avund, il existe un verbe avundas qui a la particularité d'être déponent ${ }^{17}$. La structure actancielle est la même que celle de zazdrość polonaise : att avundas någon något (à quelqu'un quelque chose), comme par exemple dans :

Han avundas sina kollegor $i$ Stockholm de många möjligheterna att utöva idrott samt den ständiga kontakten med naturen. (internet)

[il jalouse ses collègues à Stockholm (à cause de) les nombreuses possibilités de pratiquer le sport et le contact constant avec la nature]

L'expression de Y est obligatoire, quant à Z, il suffit qu'il soit récupérable dans le co(n)texte.

Missunnsamhet est jugé comme étant plus négatif du point de vue doxal qu'avund. Observons l'énoncé suivant :

Kollegerna var missunsamma därför att hon var så kompetent $i$ sitt arbete.

[Les collègues étaient jaloux parce qu'elle était si compétente dans son travail]

La traduction de missunsamm par jaloux appauvrit considérablement l'énoncé dans la mesure où le concept suédois porte en lui des présupposés d'ordre social ayant trait à l'égalitarisme déjà mentionné : tout le monde doit être plus ou moins égal et il n'est pas bon d'être mieux que les autres ${ }^{18}$. Cette idée se trouve confirmée 
par le fait que les personnes accusées de ressentir cet affect le dénient le plus souvent et s'expliquent à l'aide d'idée de justice. Missunnsamhet dans cet énoncé peut être expliqué de la manière suivante :

elle $(\mathrm{Y})$ a contrevenu à la règle d'égalité selon les collègues $(\mathrm{X})$

$\mathrm{X}$ veut que $\mathrm{Y}$ ne soit pas mieux que lui (ce n'est pas normal que $\mathrm{Y}$ le soit)

$\mathrm{X}$ veut être aussi bien que $\mathrm{Y}$.

Ces deux derniers points peuvent sembler contradictoires mais ils le sont qu'en apparence. En effet Y s'est élevé au-dessus des autres et doit retourner à sa place, c'est presque comme un retour à la normalité par punition. X peut alors s'élever. Missunnsamhet possède donc la même composante que zawiść polonaise mais s'en différencie par le fait que l'expérienceur de missunnsamhet veut posséder Z alors que l'expérienceur de zawiść ne le veut pas. On peut expliquer l'affect suédois de la manière suivante :

$X$ pense:

cette personne $Y$ a $Z$

je n'ai pas la même chose

je pense que c'est mauvais parce que cette personne n'est pas comme moi

je veux ceci : cette personne n'ait plus $Z$

je veux avoir $Z$

à cause de cela X ressent quelque chose de mauvais

Quant aux rapports entre $\mathrm{X}$ et $\mathrm{Y}, \mathrm{Y}$ doit être nécessairement d'une ou d'autre manière supérieur à $\mathrm{X}$ à travers Z. Z n'est pas nécessairement accessible à X.

Il existe le verbe missunna mais il est utilisé le plus fréquemment dans les expressions niées : att inte missunna. On emploie plus volontiers la structure essive vara missun, les actants $\mathrm{Y}$ et $\mathrm{Z}$ sont alors introduits de manière non-spécifique ou récupérables dans le contexte.

\subsection{Envy $^{19}$}

Envy anglaise est un affect qui correspond au plus près à la jalousie française, et pas du tout à l'envie comme l'étymon commun pourrait laisser croire. Envy est une réaction affective négative à la situation (Y possède Z) dans laquelle l'expérienceur souhaite être en possession de quelque chose comme Z. La différence réside peut-être dans le fait que selon la formule de Wierzbicka que nous avons déjà citée, il ne s'agit pas tant de la possession au sens matériel mais de 'quelque chose de bon qui est arrivé'. Wierzbicka ne mentionne pas de nécessité d'égalité de protagonistes ni de possibilité pour l'expérienceur d'accéder à Z, mais compte tenu de la formulation qu'elle utilise dans son explication verbale, on peut penser que ces paramètres ne sont pas contraints : 


\author{
Xpense : \\ quelque chose de bon est arrivé à cette personne \\ ce n'est pas arrivé à moi \\ c'est mauvais \\ je voudrais que de bonnes choses comme cela m'arrivent » \\ à cause de cela X ressent quelque chose de mauvais
}

Comme dans le cas des autres affects, lorsqu'on emploi le verbe envy, seul les actants X et Y sont obligatoires. $Z$ doit cependant être récupérable.

\title{
3 Les invariants et les points de variation
}

Tous les concepts encodés linguistiquement que nous avons observés dans cette présentation ont en commun le fait qu'ils concernent la situation dans laquelle un individu est en lien de possession avec quelque chose. Il s'agit pour la plupart des réponses affectives, mais l'envie française semble être une exception qui appartient au domaine volitionnel. En dehors de cette opposition affectif versus non-affectif, se profile la distinction temporelle entre les affects qui concernent une situation présente ( $\mathrm{Y}$ a $\mathrm{Z}$ ) et les affects qui concernent une situation passée ( $Y$ avait $Z$ ). La différence suivante se situe au niveau de la volonté de changement de cette situation, soit cette volonté est positive : l'expérienceur souhaite posséder quelque chose comme $Z$, soit négative est destructrice : l'expérienceur veut que Y n'ait plus Z. Un autre point de variation concerne la possibilité ou non d'accession de l'expérienceur à l'objet $\mathrm{Z}$.

On peut schématiser ces variations de la manière suivante : 


\section{Schéma 1}

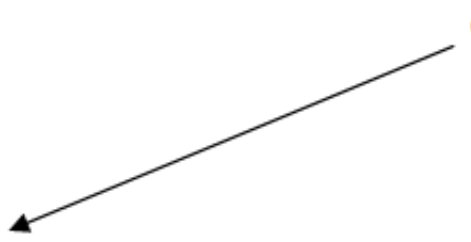

$(\mathrm{X}$ a $\mathrm{Y})$

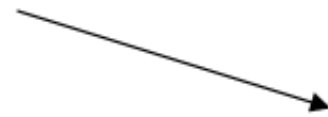

je ressens quelque chose de mauvais à cause de cela

je voudrais avoir $Z$

si j'avais Z, je ressentirais

quelque chose de bon

(je ne sais pas si je peux l'avoir)
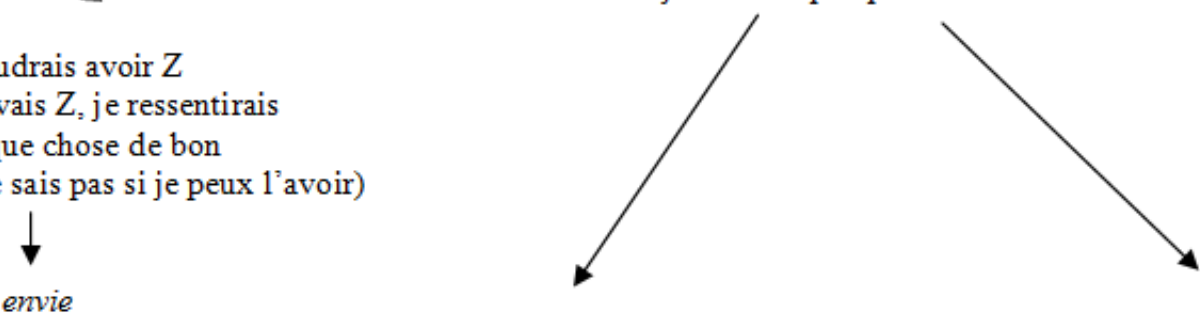

envie

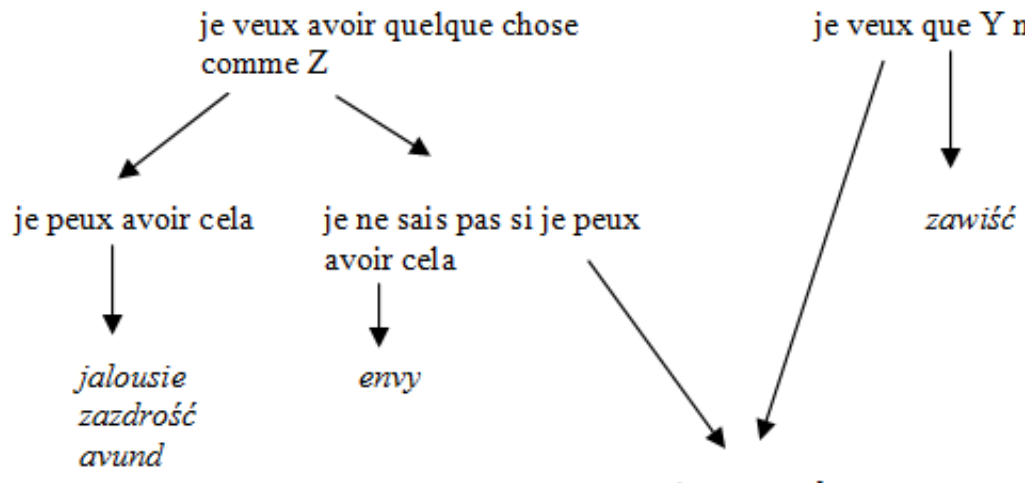

missunnsamhet

\section{Schéma 2}

(X avait Z, X n'a plus Z)

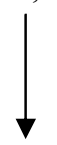

je ressens quelque chose de bon à cause de cela

Schadenfreude 
Bien évidemment il ne s'agit que d'un aperçu des différentes réactions possibles. Rappelons ici que ce n'est pas parce qu'un concept n'est pas encodé lexicalement dans une langue que les locuteurs de cette langue ne peuvent pas le ressentir. Il existe par exemple en français le concept de la joie maligne mais il est beaucoup moins saillant. Il en va de même pour le zloradstvo russe. Comme c'était prévisible, les points de variations sont peu nombreux. Mais il faudra encore élargir le corpus de réaction pour vérifier si d'autres apparaissent. Par ailleurs d'autres configurations sont théoriquement possibles, par exemple une réaction non-affective qui vise la disparition de l'objet $Z$.

Ceci pour les différences de l'ordre sémantique. En ce qui concerne les différences formelles, on peut observer les phénomènes suivants :

a) certains réactions abordées ici ont une expression expérientielle verbale : envier, zazdrościć, avundas, missunna et d'autres non; le cas suédois est remarquable dans la mesure où il s'agit d'un verbe déponent, un cas unique dans le corpus étudié ; ce fait semble important compte tenu du fait que, comme je l'ai montré (2007a), l'expression expérientielle constituée d'un verbe simple à la voix active indique un plus grand engagement de l'expérienceur dans l'affect ressenti ;

b) la structure argumentale ne s'actualise pas nécessairement dans la structure actancielle, ainsi on observe les cas suivants :

seul l'expérienceur est obligatoire, il s'agit des emplois absolus, comme je suis jaloux, ich bin Schadenfroh, l'interprétation peut être alors soit en terme de qualité, soit d'un procès affectif actualisé, mais alors les actants $\mathrm{Y}$ et $\mathrm{Z}$ doivent être soit présents dans le cotexte, soit saillants contextuellement; il s'agit toujours des expressions essives ;

dans les réactions étudiées, lorsqu'elle sont exprimées par le verbe, seul l'argument Y est obligatoirement exprimé, l'argument $\mathrm{Z}$ doit être cependant récupérable. Cette contrainte forte sur la présence de $\mathrm{Y}$ dans l'énoncé indique que $\mathrm{Z}$ ne devient intéressant qu'en tant qu'appartenant à $Y$.

On peut se demander si une telle façon d'envisager la problématique affective ne permet pas d'unir deux postulats théoriques contradictoires au sujet des affects: l'universalisme de certaines approches psychologiques (voir Christophe, 1998 pour une synthèse) et le relativisme linguistique et culturel (Harkins, Wierzbicka, 2001). La structure argumentale des affects de type de « jalousie » est toujours la même, on peut supposer qu'elle est universelle. Par contre, les variations, telles qu'elles ont été mises en évidence ici, au sein de cette structure peuvent être considérées comme linguistiquement et culturellement spécifiques. Ainsi l'universel (psychologique) est une structure, au sens que lui donne la grammaire constructionnelle (Goldberg, 1995), les différentes cultures élaborent des nuances et les encodent dans les langues qui les véhiculent, principalement au niveau sémantique.

Enfin, il reste des points intéressants à éclaircir, notamment au sujet de la jalousie et de ses équivalents. En plus de rechercher l'unité conceptuelle de différentes acceptions de ces affects, il faut se demander notamment pourquoi certaines langues comme par exemple le russe ou le suédois distinguent lexicalement entre la jalousie en tant que la convoitise d'un objet et la jalousie 'conjugale' et d'autres, comme le polonais ou le français, qui ne le font pas.

\section{Références :}

Abelson, R.P. (1981). Psychological Status of the Script Concept. American Psychologist, 36/7, 715-729.

Apresjan, J.D. (2000). Systematic lexicography. Oxford/New York: Oxford University Press.

Christophe, V. (1998). Les émotions. Tour d'horizon de principales théories. Villeneuve d'Asq : PU du Septentrion. 
Church, J. (1995). L'émotion et l'intériorisation des actions. in PAPERMAN P., OGIEN R., (eds). (1995). La couleur des pensées. Sentiments, émotions, intentions. Paris : Editions de L'Ecole des Hautes Etudes en Sciences Sociales, 219236.

Eibl-Eibesfeldt, I. (1996). Love and hate. New York: Aldine de Gruyter.

Fillmore, C. J. (2003). Form and Meaning in Language. Vol I. Papers on Semantic Roles. Stanford : CSLI Publications.

Flaux N., Van de Velde D. (2000). Les noms en français : esquisse de classement. Paris : Ophrys.

Goldberg, A. (1995). A construction Grammar Approach to Argument Structure. Chicage/London : The University of Chicago Press

Gugała, M. (2004). Pojęcie zazdrości i jego wykładniki leksykalne w języku polskim. Poradnik językowy, 6 (615), 47-58.

Harkins, J., Wierzbicka, A. (2001), Emotions in Crosslinguistic Perspective. Berlin/New York : Mouton de Gruyter.

(1999). Harrap's Universal. Dictionnaire Français-Allemand/Allemand-Français. Edinburgh/Paris/Stuttgart : Harrap's Universals.

Koselak, A. (2003). La sémantique naturelle d'Anna Wierzbicka et les enjeux interculturels. Communications, 4, pp. 8397.

Koselak, A. (2007a). Sémantique des sentiments « quand je pense à toi je ressens quelque chose de mauvais » en français et en polonais. Thèse de doctorat de l'Université Paul Verlaine de Metz. Dir. C. Masseron, A. Petitjean.

Koselak, A. (2007b). Sources et tradition polonaises en linguistique cognitive. CORELA, Numéros spéciaux, Cognition, discours, contextes. Accessible en ligne à l'URL :

http://edel.univ-poitiers.fr/corela/document.php?id=1626

Koselak, A. (à paraitre, 2008). Un modèle descriptif des affects. L'exemple de jalousie et de zazdrość. Konferencja Naukowa : Metody analizy leksykalnej : założenia teoretyczne i zastosowania praktyczne. Białystok, 9-11.10. 2008.

Koselak, A. (à paraître, 2009). Jalousie et envie : l'affectivité tout en nuances. Pratiques.

MacLean, P.D. (1980). The triune brain. in Rorty, O. A. (ed.). Explaining emotions. Berkeley/Los Angeles/London : University of California Press, 9-36.

Ortony, A., Clore, G.L., Collins, A. (1988). The Cognitive Structure of Emotions. Cambridge : Cambridge University Press.

Plantin, C. (2003). Structures verbales de l'émotion parlée. in Colletta J.M., Tcherkassof, A. (eds). Les émotions. Cognition, langage et développement, Bruxelles : Mardaga, 98-130.

Sériot, P. (2005). Oxymore ou malentendu? Le relativisme universaliste de la métalangue sémantique Naturelle d'Anna Wierzbicka. Cahiers Ferdinand de Saussure, 58, 23-43.

Wessén, E. (2003). Våra ord, deras uttal och ursprung. Stockholm: Nordstedts Ordbok.

Wierzbicka, A. (1999). Emotions across Languages and Cultures. Diversity and universals. Paris/Cambridge : Cambridge University Press/Editions de la Maison des Sciences de l'Homme.

Wierzbicka, A., Harkins, J. (2001). Introduction. in Harkins J., Wierzbicka A. (eds) 2001, Emotions in Crosslinguistic Perspective. Berlin/New York: Mouton de Gruyter, 1-34.

Zalizniak, A. (1991). De la nature du regret. $B U L A G, 17,67-75$.

${ }^{1} C f$. Koselak (2007) pour la présentation de l'introspection en tant qu'outil d'investigation. 
${ }^{2}$ J'utilise la notion de l'image linguistique du monde telle qu'elle a été élaborée par l'école ethnolinguistique de Lublin. Pour une présentation en français voir Koselak 2007a et b.

${ }^{3}$ Le lecteur pourra se rapporter par exemple à : Peeters (2002), Koselak (2003) ou au site de la métalangue sémantique naturelle : http://www.une.edu.au/lcl/nsm/

${ }^{4}$ Observons que le terme aliéniste utilisé par Plantin est lui aussi sorti d'usage, comme la conception de la pratique psychiatrique qu'il véhiculait.

${ }^{5}$ Pour une analyse de la polysémie de la jalousie $c f$. Koselak (à paraître, 2009)

${ }^{6}$ Je suis conscient que tout est discutable, j'utilise, dans ces énoncés forgés, mon acception de possibilité personnelle.

${ }^{7}$ Les explications verbales des affects données dans cette présentation sont simplifiées.

${ }^{8}$ Actuellement envie entre en collocation avec le générique nominal du domaine affectif : le sentiment, mais cela ne prouve rien dans la mesure où ce classème accepte des prédicats non affectifs. Par ailleurs, la recherche sur Google donne très peu de résultats pour les collocations avec les génériques verbaux ressentir et éprouver.

${ }^{9}$ Les lexèmes étrangers sont accordés aux déterminants français en genre selon leur genre dans la langue à laquelle ils appartiennent. Cette solution n'est pas sans problèmes parce que, dans certaines langues, comme le suédois (infra), il n'existe que les genres : neutre et non-neutre. J'utiliserai alors la forme masculine.

${ }^{10}$ Pour une comparaison détaillée de deux affects voir Koselak (à paraître, 2008).

${ }^{11}$ Les analyses détaillées de cet affect ont été effectuées dans Koselak (2007a).

${ }^{12}$ Pour mes informateurs allemands il n'y a aucun rapport entre les affects comme jalousie et Schadenfreude. Cette dernière peut être ressentie sans que quelque chose de l'ordre de jalousie ait eu lieu.

${ }^{13}$ Je tiens à remercier Elna Nilson pour son aide précieuse dans l'analyse de la problématique des affects suédois.

${ }^{14}$ Les données étymologiques proviennent de Wessén (2003).

${ }^{15}$ Il faut bien évidemment relativiser ce genre de propos pour éviter les critiques comme celles de Sériot (2005) adressées à Wierzbicka. Je ne veux pas dire que tous les scandinaves pensent et agissent de la même manière. La culture est un modèle et les gens l'intériorisent chacun à leur manière. Il n'en reste pas moins qu'il s'agit d'une tendance.

${ }^{16}$ Voir infra.

${ }^{17}$ Les verbes déponents suédois forment une classe sémantiquement hétérogène. Dans la perspective adoptée ici l'information sur la caractère déponent n'est pas suffisante, la forme passive signifie nécessairement quelque chose. Le problème semble d'autant plus complexe que, contrairement aux autres déponents, avundas accepte la forme passive alternative : bli avundad et que l'on trouve, dans l'Internet, un nombre important d'énoncés incorrects du point de vue de la norme, comme : Jag avundas av hennes mage. Dans cet exemple, la préposition $a v$ que l'on peut traduire ici par de ou par, peut être lue comme «je suis jalousé par son ventre (à elle) ». L'énoncé redevient correct (je suis jaloux de son ventre) si on supprime la préposition. Il semblerait que la forme déponente soit problématique et la morphologie passive induise les locuteurs en erreur.

${ }^{18}$ Ce genre d'égalitarisme scandinave est illustré de manière quelque peu ironique par les lois de Jante écrits par le danois, A. Sandemose, dans son roman En flygtning krydser sit spor: 1 . Tu ne dois pas croire que tu es quelqu'un! 2 . Tu ne dois pas croire que tu vaux autant que nous! 3. Tu ne dois pas croire que tu es plus malin ou plus sage que nous! 4 . Tu ne dois pas t'imaginer que tu es meilleur que nous ! 5. Tu ne dois pas croire que tu sais mieux que nous! 6 . Tu ne dois pas croire que tu es plus que nous ! 7. Tu ne dois pas croire que tu es capable de quoi que ce soit ! 8. Tu ne dois pas te moquer de nous ! 9. Tu ne dois pas croire que quelqu'un s'intéresse à toi! 10. Tu ne dois pas croire que tu peux nous apprendre quelque chose! 
${ }^{19}$ Je n'ai pas procédé à l'analyse de envy, sont rapportés ici les résultats des analyses de Wierzbicka (1999: 97 - 100). Jealousy est exclu de cette présentation parce qu'elle concerne uniquement la jalousie du type 'conjugal'. 